\title{
Electroweak measurements in the forward region at $\mathrm{LHCb}$
}
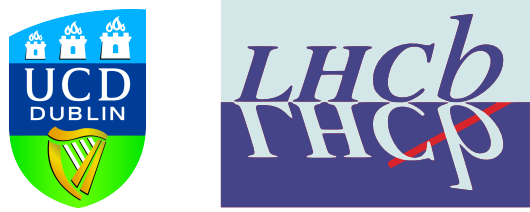

Wenchao Zhang on behalf of LHCb Collaboration

University College Dublin

Wenchao.Zhang@cern.ch

September 16-21

ISMD 2012, Kielce, Poland 


\section{Outline}

- Introduction

- LHCb Detector and Dataset

- Motivation

- Electroweak measurements
- $W \rightarrow \mu \nu_{\mu}$
- $Z \rightarrow \mu \mu$
- $Z \rightarrow e e$
- $Z \rightarrow \tau \tau$
- $\gamma^{*} / Z \rightarrow \mu \mu$

- Conclusion and outlook. 


\section{Introduction- -LHCb Detector and Dataset}

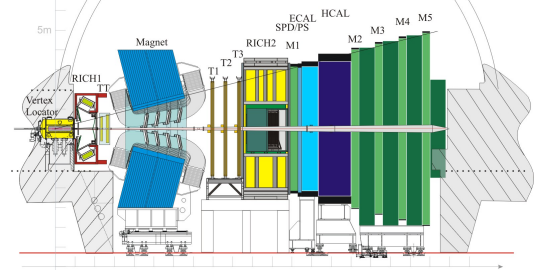

- 2010 Dataset

$$
\text { - } 37 p b^{-1}, \sqrt{S}=7 \mathrm{TeV}
$$

- 2011 Dataset

$$
\text { - } 1 \mathrm{fb}^{-1}, \sqrt{S}=7 \mathrm{TeV}
$$

- 2012 Dataset(recorded so far)

$$
\text { - } 1.35 \mathrm{fb}^{-1}, \sqrt{S}=8 \mathrm{TeV}
$$

- Single arm forward spectrometer $(2.0 \leq \eta$ $\leq 5.0$ ), designed for $B$ decays

- $2.0 \leq \eta \leq 2.5$ (common to ATLAS and CMS)

- $2.5 \leq \eta \leq 5.0$ (unique in $\mathrm{LHCb}$ )

- Precise vertex location capability(VELO)

- Tracker, ECAL, HCAL and Muon chambers (common to ATLAS and CMS)

- Two Ring Imaging Cherenkov(RICH) detectors(unique in $\mathrm{LHCb}$ )

- Complementary measurements of Electroweak Physics to ATLAS and CMS 


\section{Introduction- -Motivation}

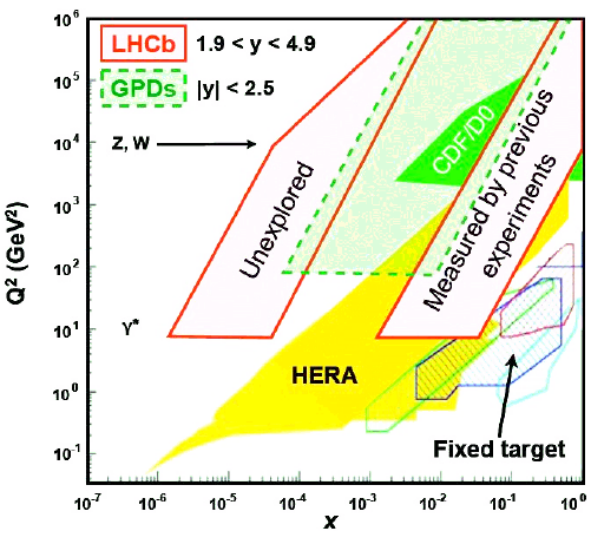

- Probe two distinct regions

- At high $\times$ region, PDFs are well constrained by previous experiments.

- At low $\times$ region, $\mathrm{LHCb}$ probes the PDFs in the region down to $x \approx 10^{-6}$

- $W$ and $Z$ probe the medium $Q^{2}(10,000 \mathrm{GeV})$ at $x \approx 5 \times 10^{-5}$

- Drell-Yan probes the low $Q^{2}(25 \mathrm{GeV})$ at $x \approx 10^{-6}$

$Q^{2}=M^{2}, x=\frac{M}{\sqrt{s}} \cdot e^{ \pm y}$ 


\section{Introduction- -Motivation}

$$
\sigma_{A B \rightarrow X}=\int d x_{a} d x_{b} \sum_{a, b} f_{a / A}\left(x_{a}, Q^{2}\right) f_{b / B}\left(x_{b}, Q^{2}\right) \sigma_{a b \rightarrow X}
$$

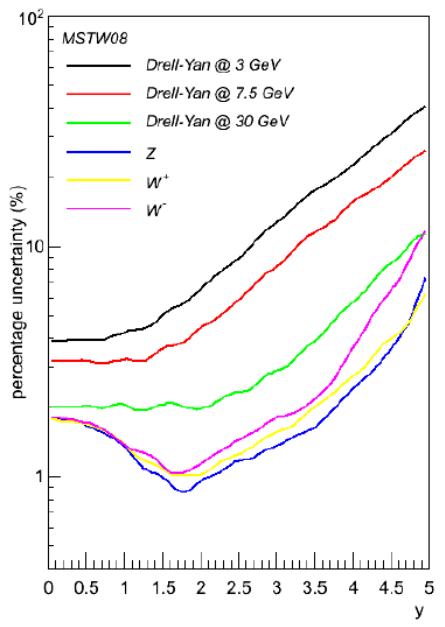

- partonic cross section uncertainty $\approx 1 \%$ at NNLO

- PDF uncertainty dominates at large rapidites $(y<2, \approx 1 \% ; y \approx 5, \approx 6-8 \%)$

- In kinematic region where PDF uncertainty is low, test SM to a precision of $1 \%$

- In kinematic region where PDF uncertainty is high, precise measurement in LHCb can constrain PDFs fits 


\section{Electroweak measurements- - $W \rightarrow \mu \nu_{\mu}$}

- Trigger on single muon $\left(p_{T}^{\mu}>10 \mathrm{GeV}\right)$

JHEP Vol.2012, No.6(2012),58

- $W \rightarrow \mu \nu_{\mu}$ candidate slection:

- track quality cuts

- $p_{T}^{\mu}>20 \mathrm{GeV}$

- $2.0<\eta_{\mu}<4.5$

- $p_{T}^{\text {extra }}<2 \mathrm{GeV}$

- unbiased IP $<40 \mu m$

- $\frac{E_{E C A L}+E_{H C A L}}{p}<0.04$

- track isolation

- charged $p_{T}^{\text {cone }}<2 \mathrm{GeV}$

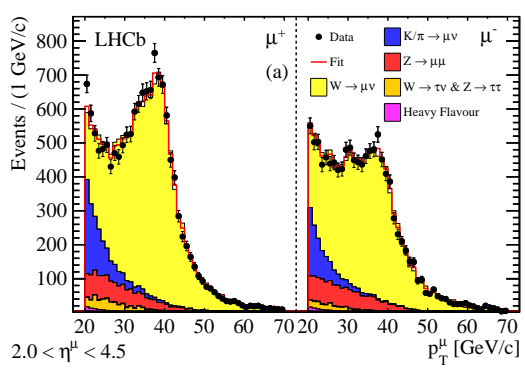

- neutral $E_{T}^{\text {cone }}<2 \mathrm{GeV}$

- $W \rightarrow \mu \nu_{\mu}$ candidate yield $\left(37 p b^{-1}\right)$

- $N_{W^{+}}: 14660, N_{W^{-}}: 11618$

- $k / \pi$ decay background

- shape taken from data

- normalisation from fit

- $Z \rightarrow \mu \mu(\tau \tau)$ and $W \rightarrow \tau \nu_{\tau}$ - shape taken from MC

- normalisation from data

- Heavy flavor

- shape taken from data with anti-cut on IP

- normalisation from Data 


\section{Electroweak measurements- $-W \rightarrow \mu \nu_{\mu}$}

$$
\sigma_{W \rightarrow \mu \nu_{\mu}}\left(p_{T}>20 \mathrm{GeV}, 2.0<\eta_{\mu}<4.5\right)=\frac{N^{W} \cdot \rho^{W} \cdot f_{F S R}^{W}}{\mathscr{L} \cdot \mathcal{A}^{W} \cdot \varepsilon_{r e c}^{W} \cdot \varepsilon_{s e l}^{W}}
$$

- $N^{W}$ : number of $W$ candidates

- $\mathcal{A}^{W}$ : determined from $\mathrm{MC}$ events

- $\rho^{W}$ : sample purity which is determined by the fit

- $\varepsilon_{r e c}^{W}=\varepsilon_{t r g}^{\mu} \cdot \varepsilon_{t r k}^{\mu} \cdot \varepsilon_{i d}^{\mu}$

- tag-probe method

- muon trigger eff. $\left(\varepsilon_{\text {trg }}^{\mu}\right)$

- muon tracking eff. $\left(\varepsilon_{\text {trk }}^{\mu}\right)$

- muon identification eff. $\left(\varepsilon_{i d}^{\mu}\right)$

- $\varepsilon_{\text {sel }}^{W}$ for selection cuts(data)

- $f_{F S R}^{W}$ correction factor for FSR

\begin{tabular}{c|c|c}
\hline Systematic & $\sigma_{W^{+} \rightarrow \mu \nu_{\mu}}[\%]$ & $\sigma_{W-\rightarrow \mu^{-} \bar{\nu}_{\mu}}[\%]$ \\
\hline Purity & \pm 1.2 & \pm 0.9 \\
Shape & \pm 0.9 & \pm 1.0 \\
$\varepsilon_{\text {rec }}$ & \pm 2.2 & \pm 2.0 \\
$\varepsilon_{\text {sel }}$ & \pm 1.8 & \pm 1.7 \\
$f_{F S R}$ & \pm 0.01 & \pm 0.02 \\
\hline Total & \pm 3.2 & \pm 2.9 \\
\hline Luminosity & \pm 3.5 & \pm 3.5 \\
\hline
\end{tabular}

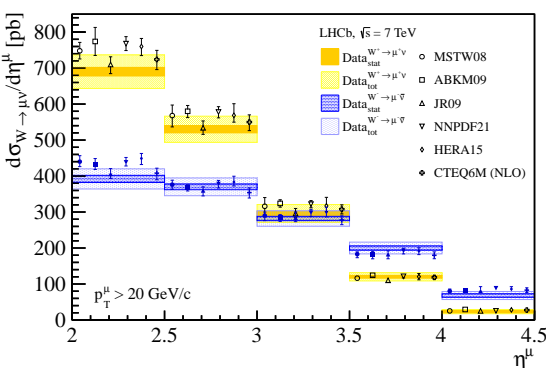




\section{Electroweak measurements- $-Z \rightarrow e e$}

- Trigger on single electron

- $p_{T}^{e}>15 \mathrm{GeV}$

- $Z \rightarrow$ ee candidate selection

- track quality cuts

- $p_{T}^{e}>20 \mathrm{GeV}$

- $2.0<\eta_{e}<4.5$

- $M_{e e}>40 \mathrm{GeV}$

- $E_{E C A L} / p>0.1$

- $E_{H C A L} / p<0.05$

- $E_{P R S}>50 \mathrm{MeV}$

- $Z \rightarrow$ ee candidate yield

- $N_{Z \rightarrow e e}=21526, N_{b k g}=473 \pm 22$

- $\mathscr{L}=945 p b^{-1}(2011)$

- misidentified pions or kaons bkg

- estimated from same sign event

- $473 \pm 22$ events
LHCb-CONF-2012-011

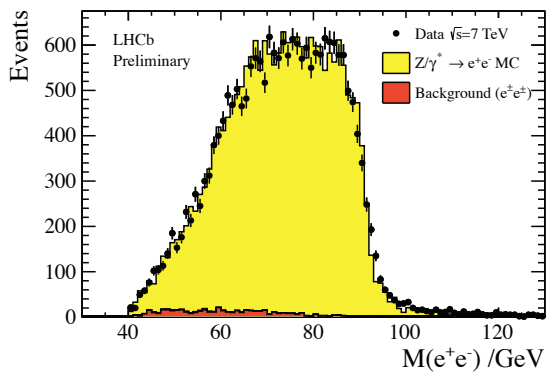

- $Z \rightarrow \tau \tau$ background

- estimated from MC

- $<20$ events

- top quark pair decay bkg

- estimated from MC

- $<20$ events 


\section{Electroweak measurements- $-Z \rightarrow e e$}

$$
\sigma_{Z \rightarrow e e}\left(p_{T}^{e}>20,2.0<\eta_{e}<4.5,60<M_{e e}<120\right)=\frac{N\left(e^{+} e^{-}\right)-N\left(e^{ \pm} e^{ \pm}\right)}{\mathscr{L} \cdot \varepsilon_{\text {rec }}^{Z} \cdot \varepsilon_{k i n}^{Z}} \cdot f_{F S R}^{Z} \cdot f_{M Z}
$$

- $\varepsilon_{\text {rec }}^{Z}=\varepsilon_{\text {trig }}^{e} \cdot\left(\varepsilon_{\text {trk }}^{e}\right)^{2} \cdot\left(\varepsilon_{\text {id }}^{e}\right)^{2}$

- $\varepsilon_{i d}^{e}$ includes the probability to associated with calorimeter info.

- $\varepsilon_{\text {kin }}^{Z}$ correction factor for bremsstralung(MC)

- $f_{F S R}^{Z}$ correction factor for FSR

- $f_{M Z}$ corrects for Drell-Yan events with true mass outside the mass range but passing event sel.(MC)

\begin{tabular}{c|c}
\hline Systematic & $\Delta \sigma_{Z \rightarrow e e}[\%]$ \\
\hline$\varepsilon_{\text {kin }}$ & \pm 1.8 \\
$\varepsilon_{\operatorname{trg}}^{e}$ & $0.4-14.0$ \\
$\varepsilon_{\operatorname{trk}}^{e}$ & $1.6-1.7$ \\
$\varepsilon_{\text {id }}^{e}$ & $0.5-2.8$ \\
$f_{\mathrm{FSR}}$ & \pm 0.1 \\
\hline Total & \pm 3.2 \\
\hline Luminosity & \pm 3.5
\end{tabular}

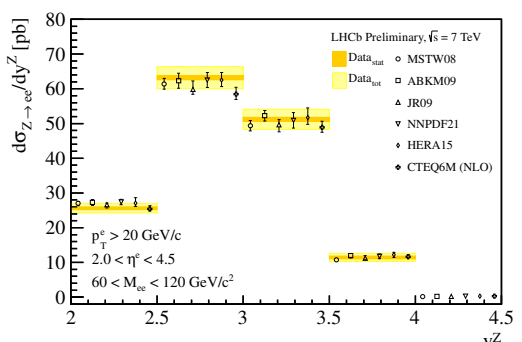




\section{Electroweak measurements- $-Z \rightarrow \tau \tau$}

- with two final states $\tau_{\mu} \tau_{\mu}$ and $\tau_{\mu} \tau_{e}$

- Trigger on single muon $\left(p_{T}^{\mu}>10 \mathrm{GeV}\right)$

- $Z \rightarrow \tau \tau$ candidate selection

- $E_{E C A L+H C A L}^{\mu} / P^{\mu}<0.2$

- $E_{E C A L}^{e} / P^{e}>0.1, E_{H C A L}^{e} / P^{e}<0.05$

- $E_{P R S}^{e}>0.05$

- $p_{T_{1}}^{\mu}>20 \mathrm{GeV}, p_{T_{2}}^{\mu / e}>5 \mathrm{GeV}$

- $2.0<\eta_{\mu / e}<4.5, M_{\mu \mu / e}>20 \mathrm{GeV}$

- $\Delta \phi_{\mu \mu / e}>2.7$ radius(back-to-back)

- Ilepton $^{\text {le }}$ (Isolation)

- $I P_{\text {sum }}$ significance $>4\left(\tau_{\mu} \tau_{\mu}\right)$

- $p_{T}^{\text {balence }}>0.2, M_{\mu \mu}<80 \mathrm{GeV}\left(\tau_{\mu} \tau_{\mu}\right)$

- $\mathscr{L}=246 p b^{-1}(2010 / 2011)$

- $Z \rightarrow \tau \tau$ candidate yield

$$
\begin{aligned}
& \text { - } N_{\text {tot }}^{\tau_{\mu} \tau_{e}}=81, N_{b k g}^{\tau_{\mu} \tau_{e}}=12.4 \pm 2.7 \\
& \text { - } N_{\text {tot }}^{\tau_{\mu} \tau_{\mu}}=33, N_{b k g}^{\tau_{\mu} \tau_{\mu}}=7.1 \pm 2.0
\end{aligned}
$$

LHCb-CONF-2011-041
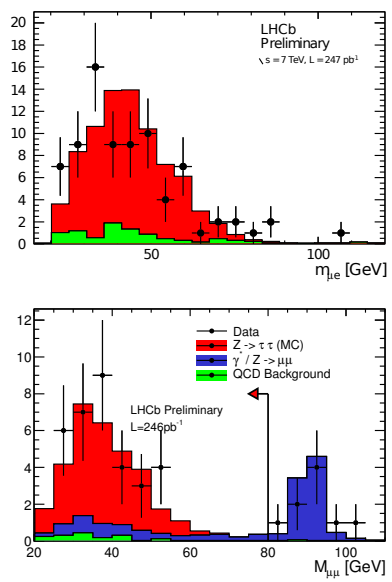

- SS QCD bkg(Data)

- $Z \rightarrow \mu \mu \operatorname{bkg}($ Data $)$ 


\section{Electroweak measurements- - $Z \rightarrow \tau \tau$}

$$
\begin{aligned}
& \sigma_{Z \rightarrow \tau_{\mu} \tau_{e}}\left(p_{T}^{\tau}>20,2.0<\eta_{\tau}<4.5,60<M_{Z}<120\right)=\frac{N^{Z}-N^{b k g}}{2 \cdot \mathscr{L}^{Z} \cdot \varepsilon_{\text {rec }}^{Z} \cdot \varepsilon_{\text {sel }}^{Z} B R\left(\tau \rightarrow e \nu_{e} \nu_{\tau}\right) \cdot B R\left(\tau \rightarrow \mu \nu_{\mu} \nu_{\tau}\right)} \\
& \sigma_{Z \rightarrow \tau_{\mu} \tau_{\mu}}\left(p_{T}^{\tau}>20,2.0<\eta_{\tau}<4.5,60<M_{Z}<120\right)=\frac{N^{Z}-N^{b k g}}{\mathscr{L} \cdot \mathcal{A}^{Z} \cdot \varepsilon_{\text {rec }}^{Z} \cdot \varepsilon_{\text {sel }}^{Z} \cdot B R\left(\tau \rightarrow \mu \nu_{\mu} \nu_{\tau}\right)^{2}}
\end{aligned}
$$

\begin{tabular}{c|cc}
\hline Systematic & $\Delta \sigma_{Z \rightarrow \tau_{\mu} \tau_{e}}[\%]$ & $\Delta \sigma_{Z \rightarrow \tau_{\mu} \tau_{\mu}}[\%]$ \\
\hline$\varepsilon_{\text {sel }}$ & \pm 6.5 & \pm 8.1 \\
$\varepsilon_{\text {trg }}^{\mu}$ & \pm 1.3 & \pm 1.3 \\
$\varepsilon_{\text {trk }}^{\mu}$ & \pm 2.4 & \pm 2.4 \\
$\varepsilon_{\text {id }}^{\mu}$ & \pm 0.2 & \pm 0.2 \\
$\varepsilon_{\text {trk }}^{e}$ & \pm 3.8 & \\
$\varepsilon_{\text {did }}^{e}$ & \pm 1.0 & \\
\hline Total & \pm 10.1 & \pm 11.2 \\
\hline Luminosity & \pm 6.0 & \pm 6.0
\end{tabular}

$$
\begin{gathered}
\sigma_{Z \rightarrow \tau_{\mu} \tau_{\mu}}=89 \pm 15_{\text {stat. }} \pm 10_{\text {syst. }} \pm 5_{\text {lumi. }} \text { pb (LHCb preliminary) } \\
\sigma_{Z \rightarrow \tau_{\mu} \tau_{e}}=79 \pm 9_{\text {stat. }} \pm 8_{\text {syst. }} \pm 4_{\text {lumi. }} \text { pb (LHCb preliminary) }
\end{gathered}
$$




\section{Electroweak measurements- $-Z \rightarrow \tau \tau$}

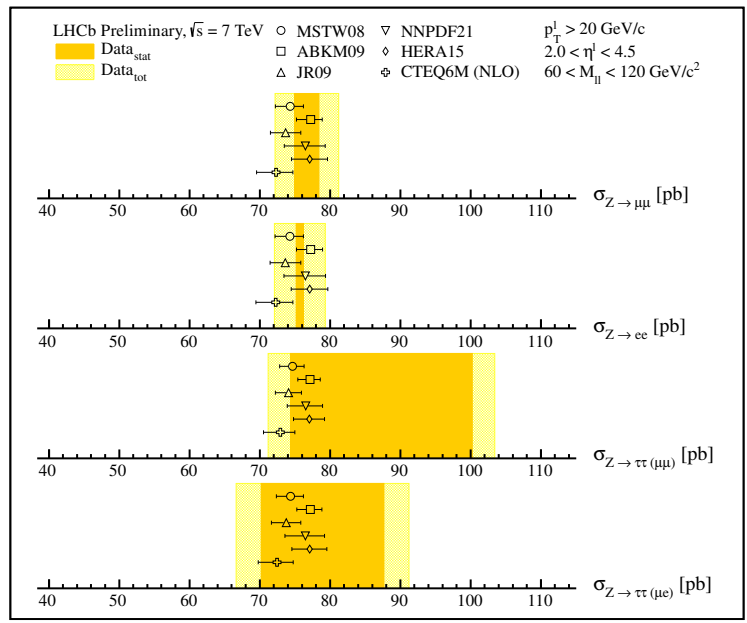

- Results are consistent among the muon, electron and tau channels.

- Lepton universality is confirmed. 


\section{Electroweak measurements- $-\gamma^{*} / Z \rightarrow \mu \mu$}

- Trigger on dimuon $\left(p_{T}^{\mu}>2.5 \mathrm{GeV}\right)$

- $\gamma^{*} / Z \rightarrow \mu \mu$ candidate selection

- track quality cuts

- $p^{\mu}>10 \mathrm{GeV}, 2.0<\eta_{\mu}<4.5$

- $p_{T}^{\mu}>3 \mathrm{GeV}$ for $M_{\mu \mu}<40 \mathrm{GeV}$

- $p_{T}^{\mu}>15 \mathrm{GeV}$ for $M_{\mu \mu}>40 \mathrm{GeV}$

- $5 \leq M_{\mu \mu} \leq 120 \mathrm{GeV}$

- $\gamma^{*} / Z \rightarrow \mu \mu$ signal yield $\left(35 p b^{-1}\right)$

- By fitting the Minlso distribution in data to template of signal and bkgs.

- Minlso defined as minimum fraction of muon-jet $p_{T}$ carried by $\mu$

- 9 mass bins, 5 rapidity bins in 2 mass regions

- Heavy flavor bkg

- shape taken from data with IP cut - free to vary in the fit
LHCb-CONF-2012-013

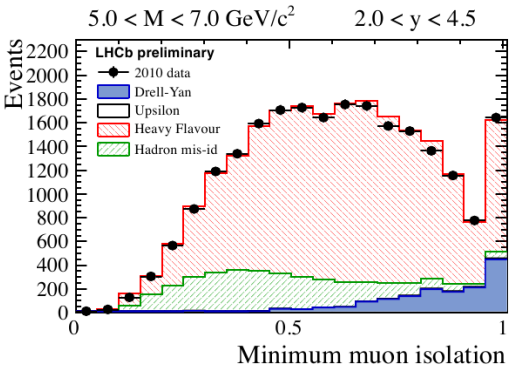

- hadron misidentification bkg

- shape taken from data

- free to vary in the fit

- upsilon decay bkg

- shape taken from MC

- normalised with $\Upsilon$ data 


\section{Electroweak measurements- $-\gamma^{*} / Z \rightarrow \mu \mu$}

$$
\sigma_{\gamma^{*} / Z \rightarrow \mu \mu}\left(p^{\mu}>10, p_{T}^{e}>3(15), 2.0<\eta_{\mu}<4.5,5<M_{\mu \mu}<120\right)=\frac{\rho}{\mathcal{A} \cdot \mathscr{L}} \sum_{i=1}^{N} \frac{1}{\varepsilon_{i}}
$$

- $\varepsilon^{\gamma^{*} / Z}=\varepsilon_{t r g}^{\mu} \cdot\left(\varepsilon_{t r k}^{\mu}\right)^{2} \cdot\left(\varepsilon_{i d}^{\mu}\right)^{2}$

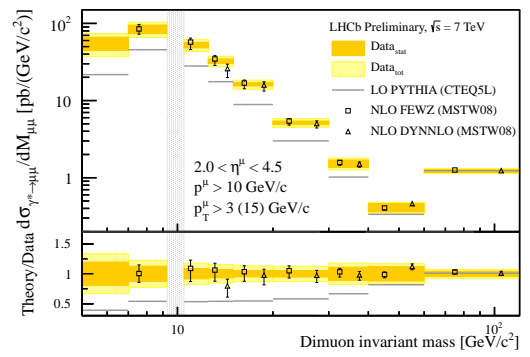

\begin{tabular}{c|c}
\hline Systematic & $\Delta \sigma_{\gamma^{*} / Z \rightarrow \mu \mu}[\%]$ \\
\hline$\varepsilon_{\operatorname{trg}}^{\mu 2}$ & $1.7-4.3$ \\
$\varepsilon_{\operatorname{trk}}^{\mu}{ }_{2}{ }^{2}$ & $4-10$ \\
$\varepsilon_{\text {id }}$ & \pm 1.4 \\
HF template & $1-24$ \\
mis-id template & $1-4$ \\
signal template & $1-8$ \\
\hline Luminosity & \pm 3.5
\end{tabular}

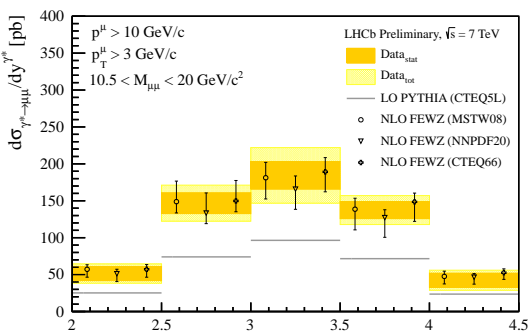

Dimuon rapidity $15 / 27$ 


\section{Conclusion and outlook}

- W, Z and Drell-Yan cross sections have been measured in different channels.

- The data results are consistent with the NNLO(NLO) theoretical predictions of different PDF sets

- Updates with full 2011 dataset are upcoming.

- Looking forward to 2012 data analysis.

- Thanks for your attention. 


\section{Backup slides}




\section{Electroweak measurements- $-W \rightarrow \mu \nu_{\mu}$}

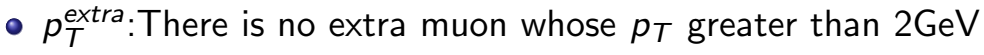

- unbiased IP: The vertex has been unbiased by excluding the candidate track from the fiting algorithm. Muons from the $\mathrm{W}$ decay have a large weight in the vertex reconstruction procedure, strongly shifting the resulting position towards them. 


\section{Electroweak measurements- - $W \rightarrow \mu \nu_{\mu}$}
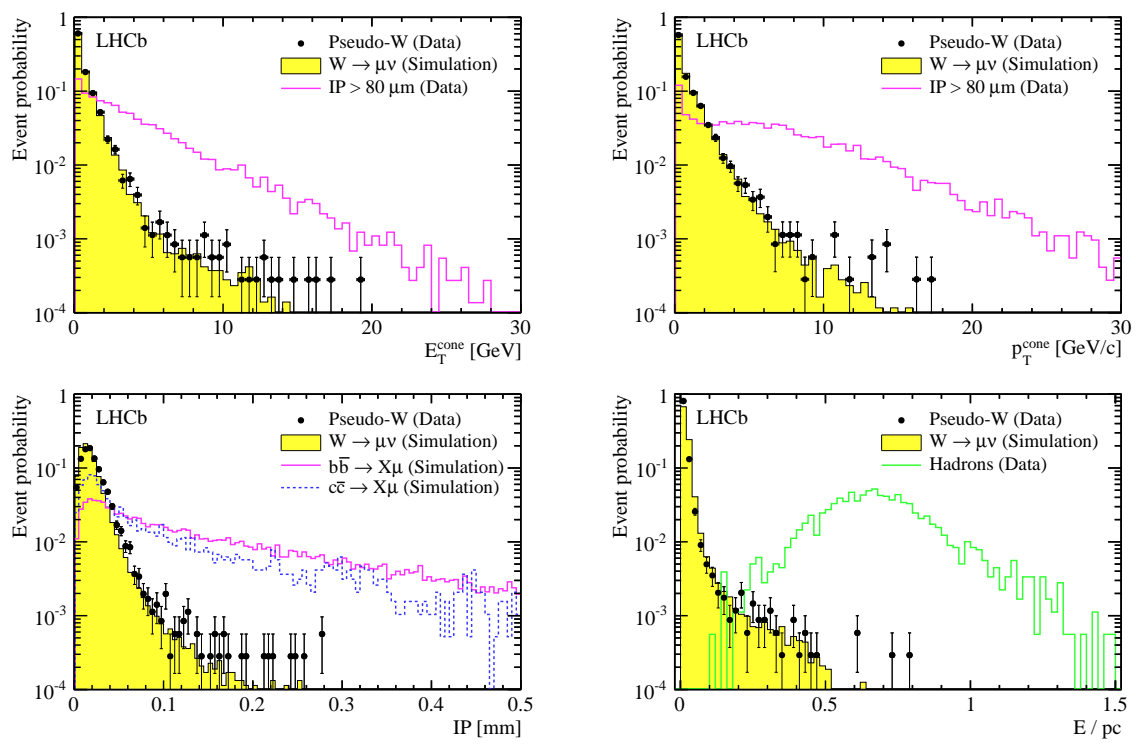

\section{Wenchao Zhang (UCD)}




\section{Electroweak measurements- - $W \rightarrow \mu \nu_{\mu}$}

- Theorectical predictions are calculated with the DYNNLO generator at NNLO(NLO) with different PDF sets.

- For MSTW08 PDF set, see Eur.Phys.J.C63 189[arXiv:0901.0002]

- For ABKM09 PDF set, see Phys.Rev.D81 014032[arXiv:0908.2766]

- For JR09 PDF set, see Phys.Rev.D79 074023[arXiv:0810.4274]

- For NNPDF2.1 PDF set, see Nucl.Phys.B838 136-206[arXiv:1002.4407]

- For HERA1.5 PDF set, see JHEP1001 109[arXiv:0911.0884]

- For CTEQ6.6 PDF set, see Phys.Rev.D78 013004[arXiv:0802.0007] 


\section{Electroweak measurements- $-Z \rightarrow \mu \mu$}

- The fit has been performed with a crystall ball function plus an exponential function.

- $Z \rightarrow \tau \tau$ background

- estimated from MC

- $0.6 \pm 0.1$ events

- heavy flavor background

- estimated from data

- $3.5 \pm 0.8$ events

- misidentified pions or kaons

- estimated from same sign event in data

- less than one event

- W pair production(MC)

- $0.2 \pm 0.1$ events

- top quark pairs decay (MC)

- $0.5 \pm 0.2$ events 


\section{Electroweak measurements- $-Z \rightarrow e e$}

- Energy recorded in individual calorimeter cells saturates at $E_{T} \approx 10 \mathrm{GeV}$

- One significant improvement to the relevant trigger lines occurred during August 2011, affecting the trigger efficiency. The data samples before and after this change were treated separately.

- The muon-jet is defined as the jet which contains the muon.

- The jet is reconstructed with the anti-kt algorithm with the size $R=\sqrt{\Delta \eta_{i j}^{2}+\Delta \phi_{i j}^{2}}=0.5$

- Here, $\Delta \eta_{i j}$ and $\Delta \phi_{i j}$ gives the separation of two particles in the jet in $\eta$ and azimuthal angle $\phi$ 


\section{Electroweak measurements- - $Z \rightarrow \tau \tau$}

- $\Delta \phi_{\mu \mu / \mathrm{e}}$ : The angle between the two leptons.

- $I^{\text {lepton }}=\frac{p^{\text {lepton }}-\sum_{i} p_{i}^{\text {track }}}{p^{\text {lepton }}+\sum_{i} p_{i}^{\text {track }}}$ where the sum extends over all charged tracks for which $\sqrt{\left(\phi^{\text {lepton }}-\phi_{i}^{\text {track }}\right)^{2}+\left(\eta^{\text {lepton }}-\eta_{i}^{\text {track }}\right)}<0.5$

- $I P_{\text {sum }}$ is the sum of the two signed impact parameters. The impact parameter of each muon is calculated as the distance of closest approach to the nearset primary vertex. It is signed according to the sign of the angular momentum of the track around the vertex.

- $p_{T}^{\text {balance }}=\frac{p_{T}^{(1)}-p_{T}^{(2)}}{p_{T}^{(1)}+p_{T}^{(2)}}$ 


\section{Electroweak measurements- - $Z \rightarrow \tau \tau$}
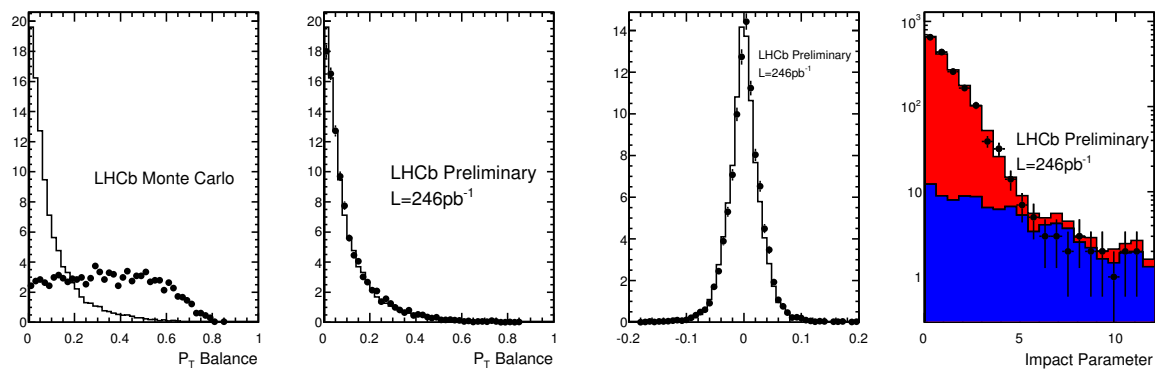

- Left

- $Z \rightarrow \tau \tau \mathrm{MC}$ (points)

- $Z \rightarrow \mu \mu \mathrm{MC}$ (histogram)

- Right

- $Z \rightarrow \tau \tau$ data(points)

- $Z \rightarrow \mu \mu \mathrm{MC}$ (histogram)

- Left

- $Z \rightarrow \mu \mu$ data(points)

- $Z \rightarrow \mu \mu \mathrm{MC}$ (histogram)

- Right

$$
\begin{aligned}
& \text { - } Z \rightarrow \tau \tau \text { data(points) } \\
& \text { - } Z \rightarrow \mu \mu \text { data(red ) } \\
& \text { - } Z \rightarrow \tau \tau \text { MC(blue) }
\end{aligned}
$$




\section{Electroweak measurements- - $Z \rightarrow \tau \tau$}
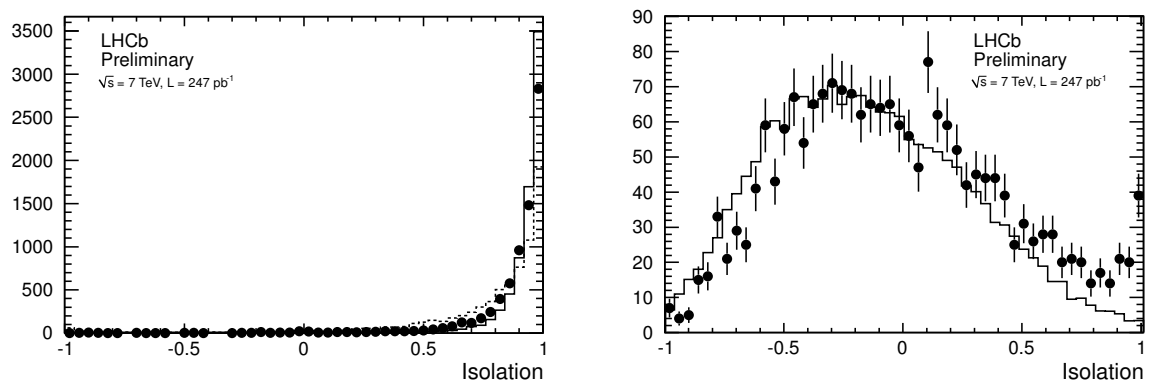

- $Z \rightarrow \mu \mu$ data(points)

- SS in $Z \rightarrow \tau_{e} \tau_{\mu}$ (points)

- $Z \rightarrow \mu \mu \mathrm{MC}$ (solid)

- $Z \rightarrow \tau_{e} \tau_{\mu} \mathrm{MC}($ dash $)$

- OS in $Z \rightarrow \tau_{e} \tau_{\mu}$ (histogram) 


\section{Electroweak measurements- $-\gamma^{*} / Z \rightarrow \mu \mu$}
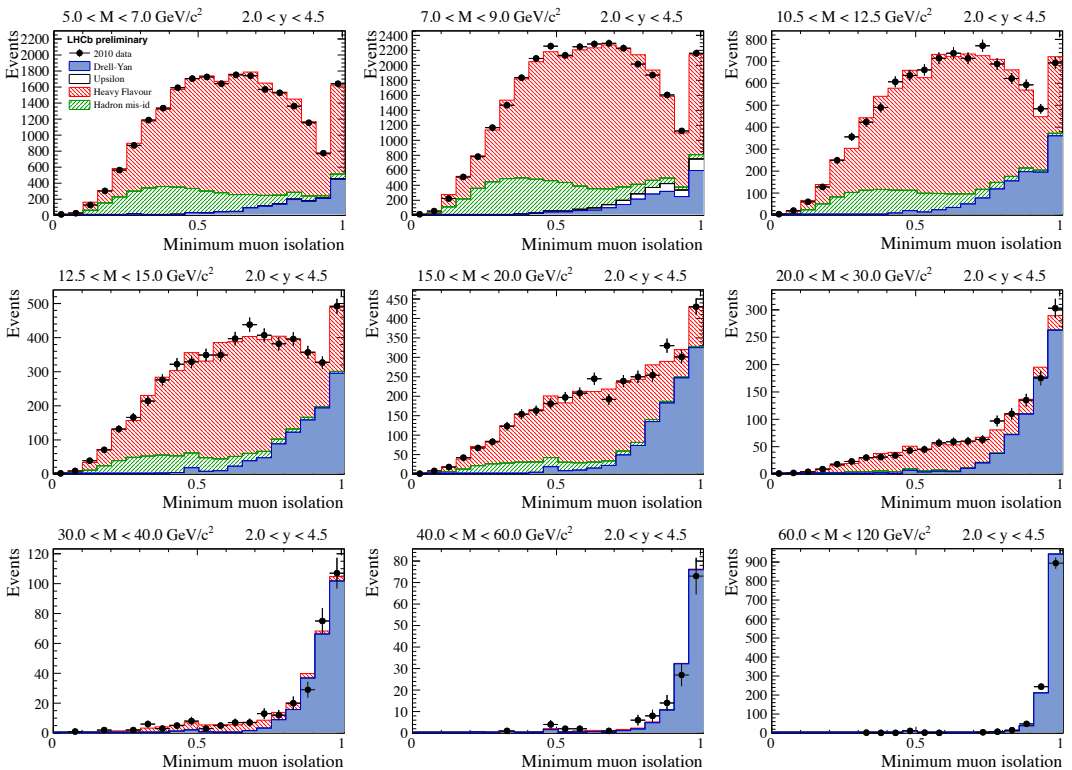


\section{Electroweak measurements- $-\gamma^{*} / Z \rightarrow \mu \mu$}
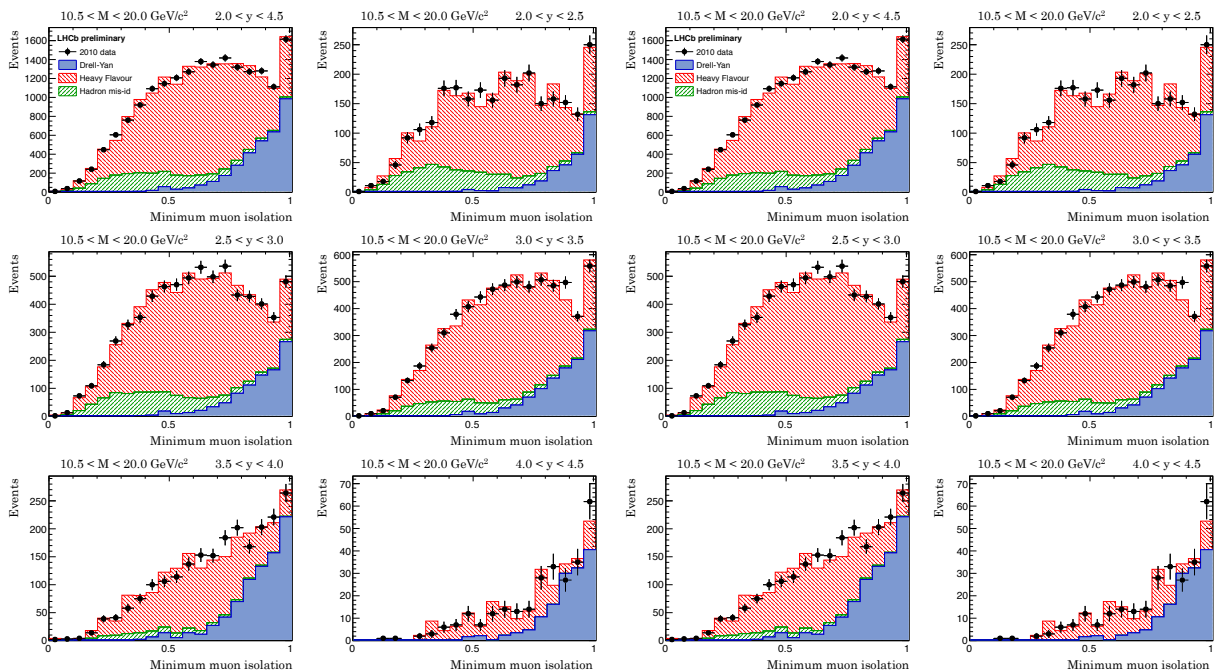

$10.5<M<20.0 \mathrm{GeV}$

$20.0<M<40.0 \mathrm{GeV}$ 